\title{
PSICOLOGIA E CUIDADOS PALIATIVOS NA ATENÇÃO PRIMÁRIA À SAÚDE: REVISÃO INTEGRATIVA
}

PSYCHOLOGY AND PALLIATIVE CARE IN PRIMARY HEALTH CARE: AN INTEGRATIVE REVIEW

PSICOLOGÍA Y CUIDADOS PALIATIVOS EN ATENCIÓN PRIMARIA DE SALUD: UNA REVISIÓN INTEGRADORA

Ana Carina Rodrigues Gois 1

Joyce Hilario Maranhão ${ }^{2}$

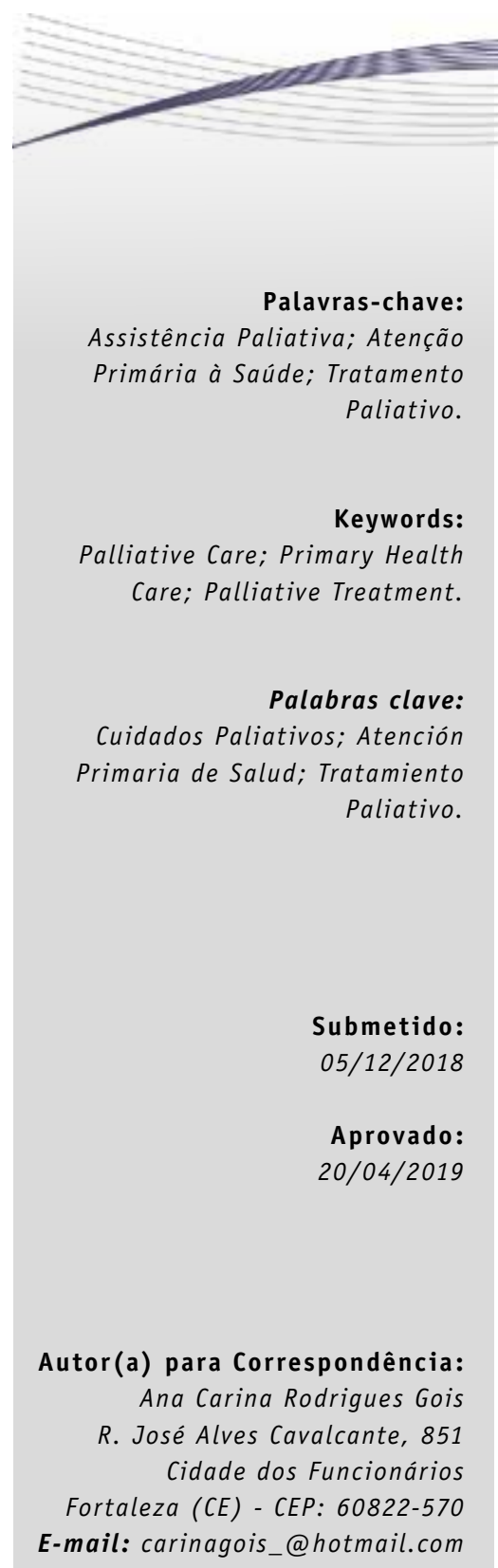

\section{RESUMO}

Este artigo descreve o conhecimento produzido na literatura acerca do cuidado paliativo na atenção primária à saúde e as possibilidades de atuação da psicologia. Realizou-se uma revisão integrativa nas bases de dados SCIELO, Lilacs, BVS Saúde e BVS Psi, por meio das palavras-chave "psicologia e cuidados paliativos", "psicologia e atenção primária" e "psicologia, cuidados paliativos e atenção primária". Incluíram-se artigos completos produzidos no Brasil em um período de 10 anos (de 2008 a 2018) e excluíram-se teses e dissertações, com base na leitura do título e resumo de todos os estudos e do texto integral dos artigos. A análise identificou 4 categorias: a) Vozes dos cuidados paliativos; b) Cuidado ao cuidador; c) Expressões de sentimentos de pacientes em cuidados paliativos; $e d$ ) Serviços de cuidados paliativos na atenção primária à saúde. A seleção final resultou em 14 artigos e evidenciou-se maior produção de estudos referentes ao cuidado ao familiar (7 artigos) e à equipe de saúde (5 artigos) - em detrimento de pesquisas voltadas aos pacientes (2 artigos). Constatou-se a necessidade de que os psicólogos desenvolvam novos estudos teóricos e práticos sobre essa modalidade de cuidado em outros tipos de serviços de saúde, tendo em vista a escassa produção científica nacional sobre o tema.

\footnotetext{
1. Psicóloga Clínica. Graduada em Psicologia pela Universidade Estadual do Ceará (UECE). Fortaleza (CE), Brasil. 2. Mestra em Saúde da Família pela Universidade Federal do Ceará (UFC). Professora de Graduação em Psicologia no UniAteneu Centro Universitário e no Centro Universitário Maurício de Nassau (UniNassau).
} 


\section{ABSTRACT}

This article describes the knowledge produced in the literature about palliative care in primary care and the possibilities of working in psychology. An integrative review was conducted in the databases SciELO, LILACS, BVS Saúde, and BVS Psi, using the keywords 'psychology and palliative care,' 'psychology and primary care,' and 'psychology, palliative care, and primary care.' Complete articles produced in Brazil within a 10-year period (from 2008 to 2018) were included and theses and dissertations were excluded, based on reading of the title and abstract of all studies and the full text of articles. The analysis identified 4 categories: a) Voices of palliative care; $b$ ) Care for the caregiver; c) Feelings expressed by patients receiving palliative care; and d) Palliative care services in primary health care. The final selection resulted in 14 articles and a higher production of studies related to family care (7 articles) and the health team (5 articles) was evidenced - to the detriment of patient-driven research (2 articles). The need for psychologists to develop new theoretical and practical studies on this modality of care in other types of health services was found, due to the scarce national literature on the theme.

\section{RESUMEN}

Este artículo describe el conocimiento producido en la literatura sobre cuidados paliativos en atención primaria y las posibilidades de actuación en psicología. Se realizó una revisión integradora en las bases de datos SciELO, LILACS, BVS Saúde y BVS Psi, mediante las palabras clave 'psicología y cuidados paliativos', 'psicología y atención primaria' y 'psicología, cuidados paliativos y atención primaria'. Se incluyeron artículos completos producidos en Brasil en un período de 10 años (de 2008 a 2018) y se excluyeron tesis y disertaciones, con base en la lectura del título y del resumen de todos los estudios y del texto integral de los artículos. El análisis identificó 4 categorías: a) Voces de los cuidados paliativos; b) Cuidado del cuidador; c) Sentimientos expresados por pacientes que reciben cuidados paliativos; y d) Servicios de cuidados paliativos en atención primaria de salud. La selección final resultó en 14 artículos y se evidenció una mayor producción de estudios relacionados con la atención familiar (7 artículos) y el equipo de salud (5 artículos) - en detrimento de investigaciones dirigidas a los pacientes (2 artículos). Se constató la necesidad de que los psicólogos desarrollen nuevos estudios teóricos y prácticos sobre esta modalidad de atención en otros tipos de servicios de salud, debido a la escasa literatura nacional sobre el tema.

\section{INTRODUÇÃ O}

A psicologia em saúde é uma especialidade que se preocupa com a inserção dos psicólogos em espaços de cuidado em saúde e com a produção da subjetividade diante de eventos de adoecimento e de tratamento de doenças agudas e crônicas. 0 especialista em psicologia em saúde atua em equipes multiprofissionais e interdisciplinares e seu trabalho visa a estabelecer estratégias de intervenção com os pacientes, com as famílias, com os profissionais da saúde, em caráter individual, e com a coletividade ${ }^{1}$.

As experiências individuais dos psicólogos nas diversas instituições de saúde pública têm criado um volume de produções que orienta a práxis profissional e oferece maior consistência para esse campo teórico. 0 s diversos estudos abordam questões referentes às áreas da ética, da bioética, dos cuidados paliativos, do atendimento em domicílio, da saúde mental, entre outras pertinentes ao trabalho do psicólogo com os demais profissionais que participam das equipes de saúde $e^{2}$.

Para a Organização Mundial da Saúde (OMS), o cuidado paliativo (CP) é uma abordagem promotora da qualidade de vida de pacientes com doenças crônicas, evolutivas e progressivas que ameaçam a continuidade da vida. Realizam-se intervenções que buscam a prevenção da dor e o alívio do sofrimento, por meio de ações que envolvem a identificação precoce, a avaliação e o tratamento da dor e de outros problemas relacionados aos aspectos físicos, sociais, psicológicos e espirituais dos pacientes ${ }^{3,4}$.

No Brasil, o Sistema Único de Saúde (SUS) almeja ser um sistema público e universal de saúde que oferece assistência de modo equânime e integral ao indivíduo e às coletividades por meio de programas e ações de prevenção, promoção, proteção e recuperação da saúde. A atenção primária à saúde (APS) é a porta de entrada para os usuários do SUS e caracteriza-se por ações desenvolvidas pelas equipes da Estratégia de Saúde da Família (ESF) e pelas equipes do Núcleo Ampliado à Saúde da Família (NASF), com o propósito de contribuir para o cuidado em saúde da comunidade local, preservando a autonomia e corresponsabilidade dos sujeitos ${ }^{5}$.

A APS é um espaço que acolhe um número mais amplo de indivíduos adoecidos. Uma das situações que podem surgir como demanda para os profissionais 
de saúde da ESF e para o NASF são as experiências de CP em domicílio, seja por doenças crônicas, a exemplo de diabetes, hipertensão, neoplasias, cardiopatias congênitas, doenças neurológicas, seja por doenças agudas com risco iminente de morte, nas quais não há possibilidade de tratamento curativo. Ainda podem surgir casos em que o processo de elaboração da perda e do luto para os familiares requer intervenções de $C P$ mesmo que o paciente não esteja mais presente ${ }^{3,6}$.

Estudos apontam que, comumente, os pacientes em CP acompanhados na APS estão em seu domicílio, acamados ou com dificuldades de locomoção. 0 acolhimento da equipe de saúde voltado a esses usuários que se encontram em CP ou a seus familiares garante uma relação humanizada, com escuta qualificada. Nessa situação, o papel de familiares e cuidadores se torna essencial para a manutenção do cuidado, junto com o da equipe de saúde responsável pelo acompanhamento da situação clínica ${ }^{7}$. A família do paciente se envolve no processo de CP e suas experiências são diferentes daquelas dos pacientes e dos profissionais da equipe de saúde ${ }^{8}$.

Indica-se que o paciente, ao se deparar com o diagnóstico de uma doença grave e sem possibilidade de cura, pode defrontar-se com sentimentos como angústia, desespero, tristeza e incerteza. Nesse momento, cabe à equipe de psicologia proporcionar um lugar de escuta, com a finalidade de ressignificar sua vida e a proximidade da morte ${ }^{8}$.

A intervenção dos profissionais de psicologia se mostra importante para garantir a integralidade do cuidado ao paciente e a qualidade de vida dos familiares e amigos. 0 cuidado do psicólogo deve facilitar as relações entre equipe, família e paciente e propiciar a comunicação eficaz, a escuta ativa, compreensiva e reflexiva das questões abordadas pelos membros envolvidos no processo ${ }^{9}$. Sob essa perspectiva, este estudo objetivou:

- Descrever o conhecimento produzido na literatura sobre o cuidado paliativo na atenção primária à saúde e as possibilidades de atuação da psicologia.

\section{METODOLOGIA}

Este estudo adotou a seguinte questão orientadora: Quais são as possibilidades de atuação da psicologia junto aos pacientes e aos familiares em cuidados paliativos na atenção primária à saúde? Tal pergunta se baseou na relevância do

\section{...cabe à equipe de psicologia proporcionar um lugar de escuta...}

profissional de psicologia no contexto da APS, com vistas a garantir a integralidade do cuidado e a qualidade de vida do paciente ${ }^{5}$.

A revisão integrativa de literatura é o método de escolha diante do objetivo proposto neste artigo. Tal metodologia se mostra expressiva devido à sua grande relevância no campo da saúde, uma vez que proporciona uma análise criteriosa da produção científica existente e sintetiza evidências de determinado tema, contribuindo para a ampliação de discussões e reflexões ${ }^{10}$. Desse modo, o referido método busca facilitar a identificação de resultados relevantes e o direcionamento de futuras pesquisas sobre a temática investigada.

A coleta de dados ocorreu em março de 2018. As palavras-chave adotadas foram: a) "psicologia e cuidados paliativos"; b) "psicologia e atenção primária"; e c) "psicologia, cuidados paliativos e atenção primária". E as bases consultadas foram: a) SciELO; b) Lilacs; c) BVS Saúde; e d) BVS Psi.

Como critério de inclusão, optou-se por artigos publicados em um período de 10 anos (de 2008 a 2018), que deveriam estar completamente disponiveis em formato eletrônico, com acesso gratuito, redigidos em língua portuguesa e versando sobre experiência no Brasil, tendo em vista que a temática aborda as necessidades da população brasileira. A seleção dos artigos se baseou na leitura de título, resumo e texto completo. Incluíram-se os estudos que retratam a atuação da psicologia nos CPs, a atuação de profissionais de saúde na APS e a percepção dos familiares e pacientes sobre os CPs. Além das teses e dissertações, excluíram-se os artigos que contemplam a temática em questão e os artigos identificados em duplicidade.

\section{RESULTADOS E DISCUSSÃO}

Aplicando os critérios da pesquisa, identificaramse 60 artigos na base SciELO; 16 artigos na base 
Lilacs; 65 artigos na base BVS Saúde; e 11 artigos na base BVS Psi - o que totaliza 152 artigos. Após a leitura dos títulos, excluíram-se os artigos que não contemplam a temática em questão, obtendo 44 publicações, como indica a Tabela 1.

Tabela 1 - Artigos identificados nas bases de dados e palavras-chave adotadas

\begin{tabular}{|lccc|}
\hline Base de dados & Palavras-chave & Encontrados & Selecionados \\
\hline SciELO & Psicologia e cuidados & 60 & 27 \\
BVS Saúde & paliativos e atenção primária à & 65 & 11 \\
BVS Psi & saúde & 11 & 5 \\
Lilacs & & 16 & 1 \\
\hline Total & & 152 & 44 \\
\hline
\end{tabular}

Fonte: Elaborado pelas autoras.

Procedeu-se a uma segunda seleção, fundamentada na leitura dos resumos dos artigos identificados. Novamente, selecionaram-se apenas os estudos que remetem à temática em questão, finalizando a seleção com 14 artigos, como registra o Quadro 1.

Quadro 1 - Sínteses das publicações incluídas na revisão integrativa.

\begin{tabular}{|c|c|c|c|c|}
\hline $\begin{array}{l}\text { Ano de } \\
\text { publicação }\end{array}$ & Autor & Titulo & $\begin{array}{l}\text { Tipo de } \\
\text { estudo }\end{array}$ & Principais conclusões \\
\hline 2008 & Silva et al. ${ }^{13}$ & $\begin{array}{c}0 \text { câncer entrou em } \\
\text { meu lar: sentimentos } \\
\text { expressos por familiares } \\
\text { de clientes }\end{array}$ & $\begin{array}{l}\text { Pesquisa } \\
\text { qualitativa }\end{array}$ & $\begin{array}{l}\text { Sentimentos vivenciados pelas } \\
\text { famílias expressam angústia, } \\
\text { tristeza, incerteza e desejo de } \\
\text { estar com o ente querido. }\end{array}$ \\
\hline 2010 & Oliveira et al. ${ }^{17}$ & $\begin{array}{l}\text { Apoio psicológico na } \\
\text { terminalidade: } \\
\text { ensinamentos para a } \\
\text { vida }\end{array}$ & $\begin{array}{l}\text { Relado de } \\
\text { experiência }\end{array}$ & $\begin{array}{l}\text { A importância do } \\
\text { acompanhamento de pacientes } \\
\text { em processo de finitude. }\end{array}$ \\
\hline 2010 & Simino et al. ${ }^{18}$ & $\begin{array}{c}\text { Acompanhamento de } \\
\text { usuários, portadores } \\
\text { de câncer, por } \\
\text { trabalhadores da saúde } \\
\text { da família }\end{array}$ & $\begin{array}{l}\text { Pesquisa } \\
\text { qualitativa }\end{array}$ & $\begin{array}{c}\text { Necessidade e possibilidades } \\
\text { nas ESF para realizar } \\
\text { acompanhamento de pacientes } \\
\text { em cuidados paliativos. }\end{array}$ \\
\hline 2010 & Sorato et al. ${ }^{14}$ & $\begin{array}{l}\text { Cuidar e ser cuidado pelo } \\
\text { grupo de apoio protege }\end{array}$ & $\begin{array}{l}\text { Pesquisa } \\
\text { descritivo- } \\
\text { qualitativa }\end{array}$ & $\begin{array}{l}\text { Benefícios que os grupos de } \\
\text { cuidadores podem gerar para } \\
\text { o cuidado de pacientes em } \\
\text { cuidados paliativos. }\end{array}$ \\
\hline 2011 & Palmeira et al. ${ }^{20}$ & $\begin{array}{c}\text { Cuidados paliativos } \\
\text { no Brasil: revisão } \\
\text { integrativa da literatura } \\
\text { científica }\end{array}$ & $\begin{array}{l}\text { Revisão } \\
\text { integrativa }\end{array}$ & $\begin{array}{l}\text { Escassa produção de artigos } \\
\text { acerca de pacientes em } \\
\text { cuidados paliativos. }\end{array}$ \\
\hline 2013 & Gorayeb et al. ${ }^{24}$ & $\begin{array}{l}\text { Psicologia na atenção } \\
\text { primária: ações e } \\
\text { reflexões em programa } \\
\text { de aprimoramento } \\
\text { profissional }\end{array}$ & $\begin{array}{l}\text { Pesquisa } \\
\text { descritivo- } \\
\text { qualitativa }\end{array}$ & $\begin{array}{c}\text { Importância de ações da } \\
\text { psicologia na atenção básica } \\
\text { ao trabalhar fatores de risco à } \\
\text { saúde da população. }\end{array}$ \\
\hline 2013 & $\begin{array}{l}\text { Combinato e } \\
\text { Martins }^{22}\end{array}$ & $\begin{array}{l}\text { (Em defesa dos) } \\
\text { Cuidados paliativos na } \\
\text { atenção primária à saúde }\end{array}$ & $\begin{array}{l}\text { Pesquisa } \\
\text { descritivo- } \\
\text { qualitativa }\end{array}$ & $\begin{array}{l}\text { Entende-se que a finalidade } \\
\text { da atividade do profissional } \\
\text { da saúde seja promover a } \\
\text { dignidade e a qualidade } \\
\text { de vida no processo de morte. }\end{array}$ \\
\hline
\end{tabular}




\begin{tabular}{|c|c|c|c|c|}
\hline $\begin{array}{l}\text { Ano de } \\
\text { publicação }\end{array}$ & Autor & Título & $\begin{array}{l}\text { Tipo de } \\
\text { estudo }\end{array}$ & Principais conclusões \\
\hline 2013 & Costa et al. ${ }^{15}$ & $\begin{array}{c}\text { Doença crônica da } \\
\text { criança: necessidades } \\
\text { familiares e a relação } \\
\text { com a Estratégia Saúde } \\
\text { da Família }\end{array}$ & $\begin{array}{l}\text { Pesquisa } \\
\text { descritivo- } \\
\text { qualitativa }\end{array}$ & $\begin{array}{c}\text { Importância do vínculo entre a } \\
\text { equipe de saúde e os usuários } \\
\text { da APS para o alívio do } \\
\text { sofrimento emocional. }\end{array}$ \\
\hline 2013 & Queiroz et al. ${ }^{16}$ & $\begin{array}{c}\text { Percepção de familiares } \\
\text { e profissionais de saúde } \\
\text { sobre os cuidados no } \\
\text { final da vida no âmbito } \\
\text { da atenção primária à } \\
\text { saúde }\end{array}$ & $\begin{array}{l}\text { Pesquisa } \\
\text { qualitativa }\end{array}$ & $\begin{array}{c}\text { Pouca melhora na qualidade de } \\
\text { vida de pacientes em cuidados } \\
\text { paliativos que estão em seu } \\
\text { domicílio. }\end{array}$ \\
\hline 2014 & Kovács ${ }^{21}$ & $\begin{array}{l}\text { A caminho da morte com } \\
\text { dignidade no século XXI }\end{array}$ & $\begin{array}{l}\text { Pesquisa } \\
\text { descritiva }\end{array}$ & $\begin{array}{l}\text { Reflexões sobre os caminhos } \\
\text { da morte no século XXI e a } \\
\text { importância de programas de } \\
\text { cuidados paliativos. }\end{array}$ \\
\hline 2015 & Marchi et al. ${ }^{25}$ & $\begin{array}{l}\text { Ser-cuidador de } \\
\text { familiar com câncer e } \\
\text { dependente: um olhar } \\
\text { para a temporalidade }\end{array}$ & $\begin{array}{l}\text { Pesquisa } \\
\text { descritiva }\end{array}$ & $\begin{array}{c}\text { As dificuldades dos familiares } \\
\text { que acompanham pacientes } \\
\text { em terminalidade podem gerar } \\
\text { adoecimento. }\end{array}$ \\
\hline 2015 & Delalibera ${ }^{11}$ & $\begin{array}{l}\text { Sobrecarga no cuidar e } \\
\text { suas repercussões nos } \\
\text { cuidadores de pacientes } \\
\text { em fim de vida: revisão } \\
\text { sistemática da literatura }\end{array}$ & $\begin{array}{c}\text { Revisão } \\
\text { sistemática }\end{array}$ & $\begin{array}{l}\text { Maiores níveis de sobrecarga } \\
\text { física e emocional de } \\
\text { cuidadores e familiares. }\end{array}$ \\
\hline 2017 & Benites et al. ${ }^{19}$ & $\begin{array}{l}\text { Significados da } \\
\text { espiritualidade para } \\
\text { pacientes com câncer em } \\
\text { cuidados paliativos }\end{array}$ & $\begin{array}{l}\text { Pesquisa } \\
\text { descritivo- } \\
\text { qualitativa }\end{array}$ & $\begin{array}{l}\text { Estar próximo da morte leva ao } \\
\text { sujeito a veneração da vida, } \\
\text { remetendo crenças pessoais e } \\
\text { busca de sentido da vida. }\end{array}$ \\
\hline 2017 & $\begin{array}{l}\text { Combinato e } \\
\text { Martins }\end{array}$ & $\begin{array}{l}\text { Necessidades da vida na } \\
\text { morte }\end{array}$ & $\begin{array}{l}\text { Pesquisa } \\
\text { descritivo- } \\
\text { qualitativa }\end{array}$ & $\begin{array}{l}\text { As necessidades dos pacientes } \\
\text { e familiares não são restritas } \\
\text { à proximidade da morte, mas } \\
\text { se referem às condições de } \\
\text { vulnerabilidade social. }\end{array}$ \\
\hline
\end{tabular}

Fonte: Elaborado pelas autoras.

Verifica-se que os temas prevalentes nas bases de dados se referem ao trabalho do psicólogo junto à Estratégia Saúde da Família (ESF) em suas diversas possibilidades de atuação e às diversas atividades destes profissionais no campo da APS. Embora consideremos que as temáticas abordadas são relevantes para a atuação do psicólogo, uma vez que remetem à realidade do trabalho na saúde pública e às demandas diante do trabalho comunitário com os usuários, percebe-se que há poucos estudos referentes ao processo de adoecimento crônico e agudo e à morte.

No Quadro 1 se observa que existe prevalência de 50\% (7 artigos) relativa à palavra-chave “psicologia e cuidados paliativos", assim como considerável déficit em publicações que enfocam a temática "psicologia, cuidados paliativos e atenção primária à saúde", com 14,2\% (2 artigos). Esse achado demonstra a escassez da literatura nacional que debate a atuação da psicologia com foco em CP na APS, apesar desse âmbito da atenção ser considerado pela legislação da saúde pública brasileira um espaço ordenador das ações e dos serviços de saúde, com o intuito de proporcionar um cuidado universal, acessível, contínuo, integral, humanizado, equânime e coerente com as necessidades da população. Os princípios dos CPs corroboram o mesmo posicionamento diante do cuidado dos indivíduos, buscando a promoção da qualidade de vida dos pacientes e de seus familiares, mediante prevenção, alívio do sofrimento, identificação precoce e tratamento da dor $r^{4}$.

Após a análise integral dos conteúdos das publicações referidas no Quadro 1, emergiram 4 categorias 
temáticas, com base na análise de conteúdo temáticocategorial: a) Vozes dos cuidados paliativos; b) Cuidado ao cuidador; c) Expressões de sentimentos de pacientes em cuidados paliativos; e d) Serviços de cuidados paliativos na atenção primária à saúde.

\section{Vozes dos cuidados paliativos}

$\mathrm{Na}$ literatura consultada, observou-se que a maior parte dos artigos aborda os sentimentos dos familiares e cuidadores de pacientes em CPs (50\%). Também se enfoca o modo como os profissionais da saúde percebem sua atuação nos CPs na APS (35\% dos artigos). Percebe-se uma limitação do número de artigos voltados ao cuidado e à percepção de como o paciente vivencia os CPS ( $15 \%$ dos artigos).

A família do paciente se apresenta ativamente no contexto do adoecimento, seja no âmbito hospitalar, seja no domiciliar (50\% dos artigos). Responsáveis por cuidados de higiene, administração de medicação, marcação de consultas, apoio emocional ao paciente, custos, logística do tratamento, entre outros fatores, os familiares tendem a apresentar sobrecarga decorrente do cuidado e do adoecimento do ente querido ${ }^{11}$. 0 s artigos analisados abordam as repercussões desse cuidado, assim como as consequências emocionais ao vivenciar a terminalidade do ente querido. Analisa-se, ainda, a criação de grupos de apoio junto aos acompanhantes, proposta pela equipe de saúde, bem como a percepção desse grupo sobre o serviço oferecido pela APS ${ }^{12}$.

No mais, esse achado apresenta considerável distanciamento das pesquisas da abordagem dos sentimentos do paciente nos CPs - esse sujeito deveria ser o foco principal no contexto envolvido. Percebese que esse número pouco expressivo pode deverse ao diagnóstico tardio de doenças ameaçadoras à vida, o que justificaria o pouco contato com os pacientes, tendo em vista que podem encontrar-se em processo de finitude avançado, com dificuldades de locomoção e verbalização e reduzidos níveis de consciência e lucidez, o que inviabiliza a realização de entrevistas. Tal escassez também pode decorrer de CPs tardios, uma vez que, às vezes, o paciente é diagnosticado com uma doença crônica e submete-se a procedimentos invasivos, sem encaminhamento ao serviço de CPs.

Ademais, o enfoque nos profissionais da saúde é destacado nas publicações identificadas. A percepção da equipe de saúde sobre sua atuação, a dificuldade de trabalhar com a iminência da morte do ...atenção dos profissionais para com eles contribui para melhorar sua saúde mental e física...

paciente, a importância do vínculo com a família e o entrave para se comunicar com os demais níveis de atenção são as principais considerações observadas ${ }^{8}$. Desse modo, como debatido na Política Nacional de Atenção Básica $(P N A B)^{5}$, evidencia-se a importância da integração entre as ações e os serviços da rede de saúde pública, com suas diversas tecnologias que proporcionam bem-estar e qualidade de vida.

\section{Cuidado ao cuidador}

Dentre os artigos analisados, $50 \%$ enfocam os familiares. Após a leitura e análise de seu conteúdo, revelou-se que os principais sentimentos expressos pelos familiares são de incerteza, angústia e tristeza, bem como de esperança e desejo de estar com o ente querido ${ }^{12}$. Assim, o familiar que assume o papel de principal cuidador de pacientes com doenças ameaçadoras à vida apresenta maior risco de sobrecarga emocional e mobilidade física ${ }^{13}$.

Evidenciou-se, ainda, a importância de grupo de apoio aos cuidadores, seja no âmbito hospitalar ou na APS, tendo em vista que podem propiciar a elaboração de sentimentos e sensações por parte dos familiares e a valorização da família nos momentos difíceis, além de auxiliar a propagação de informações e orientações sobre o cuidado ao paciente ${ }^{14}$.

Observaram-se alterações nos diferentes aspectos da vida do cuidador durante o adoecimento do ente querido, como abandono de emprego e distanciamento de atividades de lazer e de outros familiares. A depender da estrutura funcional da família, podem existir fatores que tendem a ser complicadores ou facilitadores do processo de cuidado ${ }^{8}$.

Em 28,6\% dos artigos analisados, os cuidadores também destacaram que a atenção dos profissionais para com eles contribui para melhorar sua saúde mental e física ${ }^{14}$. Na assistência domiciliar, a equipe de saúde deve buscar a colaboração da família e trabalhar em parceria com a comunidade. Em 
$14,3 \%$ dos artigos analisados a percepção familiar quanto à ESF é atrelada ao curso da doença e ao processo de vinculação com a equipe ${ }^{15}$. A boa relação entre profissionais e familiares é essencial para a construção de um cuidado em consonância com os diversos saberes, proporcionando bem-estar aos envolvidos - isso é considerado um facilitador, já que os envolvidos são informados sobre o caso clínico e recebem apoio e informações acerca do trabalho desempenhado ${ }^{16}$.

\section{Expressões de sentimentos de pacientes em cuidados paliativos}

Apenas $28,2 \%$ dos artigos contemplaram essa temática. As publicações analisadas destacam a importância do atendimento psicológico ao paciente em processo de finitude e a elaboração de experiências emocionais vivenciadas por ele antes da morte ${ }^{17}$. Ao receber o diagnóstico de uma doença grave e ao se deparar com os CPs, o paciente pode apresentar sentimentos diversos que necessitam ser elaborados ${ }^{12}$. Vale destacar que cada sofrimento é singular, não há um protocolo ou manual sobre o modo como o paciente pode reagir diante de tal situação. Assim, mostra-se a relevância dos CPs se basearem em princípios ${ }^{4}$. Evidenciou-se um atendimento psicológico com elaboração de experiências emocionais vivenciadas pelo paciente durante o processo de finitude. Dessa forma, analisaram-se as contribuições que tal atendimento proporcionou para o paciente e a relação dele com a equipe de saúde ${ }^{17}$.

Ressalta-se a relevância das intervenções psicológicas nos fatores de risco à saúde da população atendida na rede de saúde. É papel da equipe de psicologia do serviço oferecer um lugar de escuta, com a finalidade de ressignificar a vida do sujeito e a proximidade de sua morte ${ }^{12}$. Também se faz necessário proporcionar autonomia na tomada de decisões e voz ativa durante o tratamento da dor ${ }^{18}$.

Revela-se nos artigos que o atendimento aos usuários da APS tem possibilitado enfocar o cuidado emocional e biológico da doença, porém, por vezes, a equipe de saúde que presta assistência tende a destacar o apoio ao paciente nas esferas psicológica, biológica e social, mas isso diminui a importância de contemplar a dimensão espiritual do paciente ${ }^{18}$. Assim, os artigos consideram que o conhecimento das especificidades da dimensão espiritual na interface com a unidade de cuidado pode aprimorar as práticas

\author{
...cada \\ sofrimento \\ é singular, não \\ há um protocolo \\ ou manual...
}

de humanização e atenção integral ao paciente com uma doença que ameaça a continuidade de sua vida ${ }^{19}$.

\section{Serviços de cuidados paliativos na atenção primária à saúde}

Em relação à importância de efetivo desenvolvimento de programas de CPs no Brasil, assim como à compreensão da morte com dignidade, constatou-se considerável demanda de usuários na APS diagnosticados com doenças crônicas que ameaçam a continuidade de sua vida e que necessitam da assistência integral por parte de equipe multidisciplinar6. No entanto, observou-se nos artigos que, às vezes, a equipe de saúde não detém o conhecimento necessário para avaliar tais usuários e proporcionar acompanhamento especializado e individualizado para eles e seus familiares ${ }^{9}$.

Essa carência de conhecimento técnico que fundamenta a prática de cuidado em saúde se reflete na escassez de estudos em CPs no Brasil ${ }^{20}$. Com frequência, os estudos identificados enfatizam os CPs em oncologia $(35,7 \%)$, o que compromete o conhecimento sobre outros tipos de doença que demandam atenção paliativa, como cardiopatias, doenças neuromusculares e doenças crônicas não transmissiveis ${ }^{21}$. Constata-se a necessidade de desenvolver estudos sobre a atuação em diferentes áreas e a articulação entre elas, com a finalidade de aprimorar e intensificar a discussão sobre CPs em domicílio, no ambulatório e na APS, considerando a perspectiva das diversas profissões em saúde, dos usuários e de seus familiares.

Aposta-se que a implementação de serviços de CPs na APS, considerando as necessidades e a realidade sanitária e social dos usuários, é relevante na abordagem da perda, do luto e do processo de morte e morrer ${ }^{22}$. Ademais, nas capacitações dos estudantes universitários e dos profissionais da saúde já inseridos na rede pública, não se observa estímulo à construção de espaços onde se busque 
ampla reflexão entre os profissionais e a população - tal como propõe a educação permanente em saúde ${ }^{23}$.

Ressalta-se que a oferta de CP nos serviços de saúde depende de uma postura bioética dos profissionais da saúde e de uma postura política em defesa de uma saúde pública que acolha as demandas da população e a singularidade de cada um diante de questões complexas, como o processo de adoecimento e morte, além de uma formação em saúde qualificada, interdisciplinar e interprofissional².

\section{CONCLUSÃO}

Verifica-se que tanto o conhecimento acerca dos CPs na APS produzido na literatura no período de 10 anos (de 2008 a 2018) quanto as possibilidades de atuação da psicologia apontam uma baixa produção sobre a prática dos CPs na APS e, especificamente, quanto às contribuições da psicologia com essa abordagem. Assim, urge a necessidade de que essas temáticas sejam estudadas por psicólogos na saúde pública brasileira.

Ademais, observa-se que tal achado sinaliza a necessidade de maior investimento em estudos no campo acadêmico sobre a atuação dos profissionais da saúde nos CPs na APS. No que concerne ao profissional de psicologia, pontua-se que a escassa produção científica pode relacionar-se a certa dificuldade dos psicólogos para descrever e refletir sobre seu trabalho no âmbito dos serviços de CPs e sua possivel resistência para fazer parte de equipe multiprofissional de tais serviços.

Concluiu-se ser necessário desenvolver novos estudos sobre a abordagem dos CPs em outros tipos de serviço na rede de saúde pública e é indispensável que os psicólogos exponham suas contribuições teóricas e práticas nessa modalidade de atenção à saúde.

\section{CONTRIBUIÇÃO DAS AUTORAS}

Ana Carina Rodrigues Gois contribuiu com a realização da pesquisa, o delineamento do estudo e a redação do manuscrito. Joyce Hilario Maranhão contribuiu com a redação e revisão crítica do manuscrito.

\section{REFERÊNCIAS}

1. Conselho Federal de Psicologia. Resolução n. 03/2016. Altera a Resolução CFP n. 013/2007, que institui a Consolidação das Resoluções relativas ao Título Profissional de Especialista em Psicologia e dispõe sobre normas e procedimentos para seu registro [document on the internet]. 2016 [cited 2018 Mar 4]. Available from: https:// site.cfp.org.br/wp-content/uploads/2016/04/ Resolu\%C3\%A7\%C3\%A30-003-2016.pdf

2. Souza ARB. 0 fazer do psicólogo na saúde. Cadernos de Graduação: Ciências Biológicas e da Saúde Fits [serial on the internet]. 2013 [cited 2019 May 24];1(2):79-87. Available from: https:// periodicos.set.edu.br/index.php/fitsbiosaude/ article/viewFile/620/368

3. Kubler-Ross E. Sobre a morte e o morrer: o que os doentes têm para ensinar a médicos, enfermeiras, religiosos e aos seus próprios parentes. São Paulo: Martins Fontes; 1996.

4. World Health Organization. Definition of palliative care [document on the internet]. 2017 [cited 2019 May 24]. Available from: http://www. who.int/cancer/palliative/definition/en/

5. Brasil. Portaria n. 2436, de 21 de setembro de 2017. Aprova a Política Nacional de Atenção Básica, estabelecendo a revisão de diretrizes para a organização da Atenção Básica, no âmbito do Sistema Único de Saúde (SUS) [document on the internet]. 2017 [cited 2018 Feb 28]. Available from: http:// bvsms.saude.gov.br/bvs/saudelegis/gm/2017/ prt2436 2209 2017.html

6. Marcucci FCI, Perilla AB, Brun MM, Cabrera APS. Identificação de pacientes com indicação de cuidados paliativos na Estratégia Saúde da Família: estudo exploratório. Cad Saúde Colet [serial on the internet]. 2016 [cited 2019 May 24];24(2):145-52. Available from: http://www.scielo.br/pdf/cadsc/ v24n2/1414-462X-cadsc-24-2-145.pdf

7. Barros MMAF, Mendes MLC, Frota LMA, Almeida JRS. Acolhimento em unidade de atenção primária à saúde: potencialidades e desafios. Sanare (Sobral, Online) [serial on the internet]. 2018 [cited 2019 May 18];13(2):114-9. Available from: https://sanare. emnuvens.com.br/sanare/article/view/1269/677

8. Franco MHP. Luto em cuidados paliativos. São Paulo: Conselho Regional de Medicina do Estado de São Paulo; 2008.

9. Silva MLS. 0 papel do profissional da atenção primária à saúde em cuidados paliativos. Rev Bras Med Fam Comunidade [serial on the internet]. 2014 [cited 2019 May 24];9(30):45-53. Available from: https://www.rbmfc.org.br/rbmfc/article/ view $/ 718 / 595$ 
10. Soares CB, Hogo LAK, Peduzzi M, Sangaleti C, Yonekura T. Revisão integrativa: conceitos e métodos utilizados na enfermagem. Rev Esc Enferm USP [serial on the internet]. 2014 [cited 2019 May 24];48(2):335-45. Available from: http:// www.scielo.br/pdf/reeusp/v48n2/pt 0080-6234reeusp-48-02-335.pdf

11. Delalibera M. Sobrecarga no cuidar e suas repercussões nos cuidadores de pacientes em fim de vida: revisão sistemática da literatura. Ciênc Saúde Colet [serial on the internet]. 2015 [cited 2019 May 24];20(9):2731-47. Available from: http://www. scielo.br/pdf/csc/v20n9/1413-8123-csc-20-09-2731. $\underline{\mathrm{pdf}}$

12. Combinato DS, Martins STF. Necessidades da vida na morte. Interface Comun Saúde Educ [serial on the internet]. 2017 [cited 2019 May 24];21(63):86980. Available from: http://www.scielo.br/pdf/icse/ v21n63/1807-5762-icse-1807-576220160649.pdf

13. Silva MRB, Borgognoni K, Rorato C, Morelli S, Silva MRV, Sales CA. O câncer entrou em meu lar: sentimentos expressos por familiares de clientes. Rev Enferm UERJ. 2008;16(1):70-5.

14. Sorato DB, Peres SV, Mitsuyuki MC. Cuidar e ser cuidado pelo grupo de apoio protege. Psicol. Estud [serial on the internet]. 2010 [cited 2019 May 24];15(4):751-9. Available from: http://www.scielo. $\mathrm{br} / \mathrm{pdf} / \mathrm{pe} / \mathrm{v} 15 \mathrm{n} 4 / \mathrm{v} 15 \mathrm{n} 4 \mathrm{a} 10 . \mathrm{pdf}$

15. Costa EA0, Dupas G, Sousa EFR, Wernet M. Doença crônica da criança: necessidades familiares e a relação com a Estratégia Saúde da Família. Rev Gaúch Enferm [serial on the internet]. 2013 [cited 2019 May 24];34(3):72-8. Available from: http:// www.scielo.br/pdf/rgenf/v34n3/a09v34n3.pdf

16. Queiroz AHAB, Pontes RJS, Souza AMA, Rodrigues TB. Percepção de familiares e profissionais de saúde sobre os cuidados no final da vida no âmbito da atenção primária à saúde. Ciênc Saúde Colet [serial on the internet]. 2013 [cited 2019 May 24];18(9):261523. Available from: http://www.scielo.br/pdf/csc/ v18n9/v18n9a16.pdf

17. Oliveira EA, Santos MA, Mastropietro AP. Apoio psicológico na terminalidade: ensinamentos para a vida. Psicol Estud [serial on the internet]. 2010 [cited 2019 May 24];15(2):235-44. Available from: http://www.scielo.br/pdf/pe/v15n2/a02v15n2.pdf

18. Simino GPR, Santos CB, Mishima SM. Acompanhamento de usuários, portadores de câncer, por trabalhadores da Saúde da Família. Rev Latinoam Enferm [serial on the internet]. 2010 [cited 2019 May 24];18(5):17-26. Available from: http://www. scielo.br/pdf/rlae/v18n5/pt 04.pdf

19. Benites AC, Neme CMB, Santos MA. Significados da espiritualidade para pacientes com câncer em cuidados paliativos. Estud Psicol [serial on the internet]. 2017 [cited 2019 May 24];34(2):269-79. Available from: http://www.scielo.br/pdf/estpsi/ v34n2/0103-166X-estpsi-34-02-00269.pdf

20. Palmeira HM, Scorsolini-Comin F, Peres RS. Cuidados paliativos no Brasil: revisão integrativa da literatura científica. Aletheia [serial on the internet]. 2011 [cited 2019 May 24];35(36):179-89. Available from: http://pepsic.bvsalud.org/scielo.php?script=sci ar ttext\&pid=S1413-03942011000200014

21. Kovács MJ. A caminho da morte com dignidade no século XXI. Rev Bioét [serial on the internet]. 2014 [cited 2019 May 24];22(1):94-104. Available from: http://www.scielo.br/pdf/bioet/v22n1/a11v22n1. $\mathrm{pdf}$

22. Combinato DS, Martins STF. (Em defesa dos) cuidados paliativos na atenção primária à saúde. Mundo Saúde [serial on the internet]. 2013 [cited 2019 May 24];36(3):433-41. Available from: http:// www.saocamilo-sp.br/pdf/mundo saude/95/5.pdf

23. Brasil. Portaria n. 198/GM/MS, de 13 de fevereiro de 2004. Institui a Política Nacional de Educação permanente em saúde [document on the internet]. 2004 [cited 2018 Feb 28]. Available from: http:// bvsms.saude.gov.br/bvs/saudelegis/gm/2017/ MatrizesConsolidacao/comum/13150.html

24. Gorayeb R, Borges CD, Oliveira CM. Psicologia na atenção primária: ações e reflexões em programa de aprimoramento profissional. Psicol. cienc. prof. 2012;32(3):674-685 Available from: http://www. scielo.br/pdf/pcp/v32n3/v32n3a12.pdf

25. Marchi JA, Carreira L, Sales CA. Ser-cuidados de familiar com câncer e dependente: um olhar para a temporalidade. Rev Eletr Enf. 2015;17(3). Available from: https://www.fen.ufg.br/revista/v17/n3/pdf/ v17n3a15.pdf
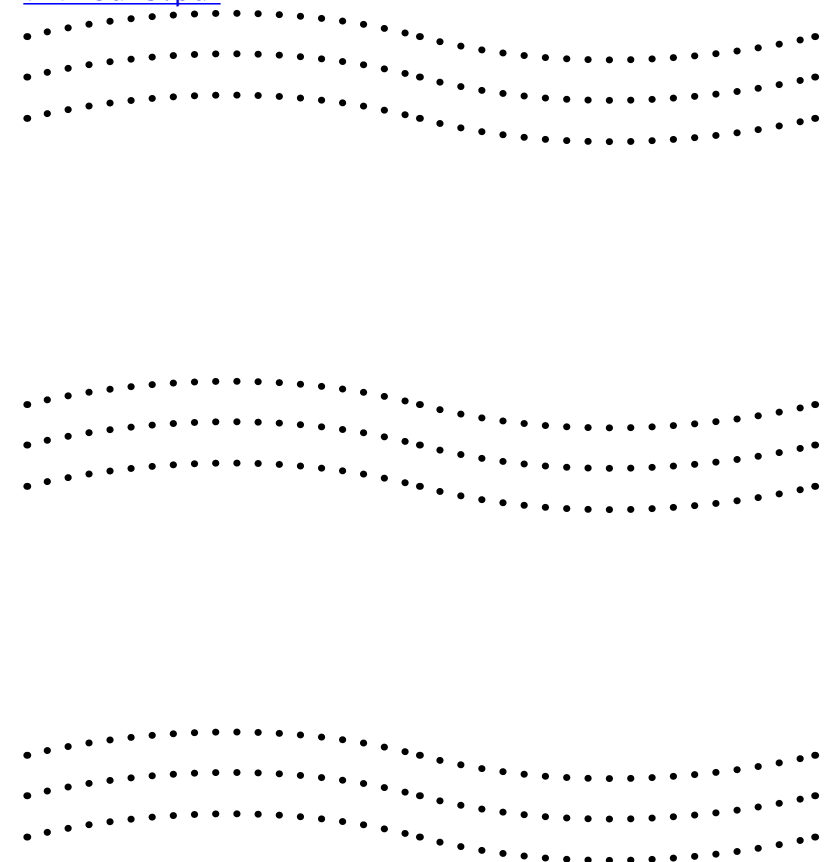